ARTÍCULO ORIGINAL

Recibido:

19/05/2019

Aprobado:

29/11/2019

\title{
Determinación de factores
} que afectan la medición de los niveles de ruido y aislamiento acústico en una cabina insonorizada para autopartes Determination of factors that affect the measurement of noise levels and sound insulation in a soundproof cabin for auto parts

\section{RESUMEN}

En el proceso de pruebas finales de vehículos, las ensambladoras de autos realizan verificaciones de ruidos internos no deseados, para luego eliminarlos, con el objeto de garantizar la satisfacción del cliente. Estas pruebas se realizan al cien por ciento de las unidades. Las mediciones de ruidos que desarrollan las ensambladoras son subjetivas y dependen de la capacidad auditiva del operario que hace la prueba. Todas las unidades que son detectadas por el cliente son segregadas y rechazadas. En el caso de que el porcentaje de unidades que tengan niveles de ruido sobre el estándar del cliente se puede llegar a embarques controlados, que implican inspecciones adicionales, a costo del proveedor, en sus instalaciones. Mediante el uso de una cabina insonorizada, se realiza la detección de los productos que no cumplen el estándar del cliente, en la propia planta del proveedor, sin ser necesario montar la parte con ruido en el vehículo. Sin embargo, para poder simular un proceso similar al que se tiene en un vehículo, se deben determinar los factores que tienen impacto en la medición del ruido. En este proyecto, no se construyó la cabina insonorizada, sino que se la simuló con un programa informático y cálculos con un modelo matemático validado de aislamiento acústico para paredes dobles, y mediante el uso de la metodología del diseño de experimentos, se simularon en el software cada uno de los factores. A fin de determinar la combinación de variables, se usó un diseño matricial, con la ayuda del software estadístico. Se usaron en la simulación materiales que por su costo pueden encontrarse fácilmente en el mercado ecuatoriano, para que luego este dispositivo pueda usarse en todas las plantas de producción de autopartes, permitiendo así que se valide el nivel de ruido. Los resultados de la simulación se compararon con resultados de varios autores que han estudiado el aislamiento de acústico de paneles sándwich con y sin absorbente en el medio. Finalmente, con esta metodología se llegaron a determinar los factores que influyen en la medición de ruidos en una cabina insonorizada de bajo costo con el objeto de reducir el ruido aéreo del ambiente de medición.

\section{Palabras Clave:}

Vibración; cabina a prueba de ruido; niveles de ruidos; aislamiento acústico.

\section{ABSTRACT}

At the final vehicle testing process, car assemblers perform checks of unwanted internal noise, and then eliminate them, in order to guarantee customer satisfaction. These tests are carried outatone hundred percent of the units. The noise measurements made by the assemblers are subjective and depend on hearing ability of the injector performing the test. All the units that are detected by the client are segregated and rejected. In the event that the percentage of units that have noise levels over the customer's standard, controlled shipments can be reached, which imply additional inspections, at the supplier's expense, at their facilities. Through the use of a soundproof chamber, the detection of products that do not meet the customer's standard is performed in the supplier's own plant, without the need to mount the part with noise in the vehicle. However, in order to simulate a process similar to that in a vehicle, factors that have an impact on noise measurement must be determined. In this project, the soundproof chamber was not built, but it was simulated with a computer program and calculations with a validated mathematical model of acoustic insulation for double walls, and by using the methodology of the design of experiments, they were simulated in the software each of the factors. To determine the combination of variables, a matrix design was used, with the help of statistical software. Materials were used in the simulation, which, due to their cost, can easily be found in the Ecuadorian 
market, so that this device can then be used in all the production plants of auto parts, so that the noise level is validated. The results of simulation were compared with the results from several authors than have studied the transmission loss in sandwich panel with and without absorbent in the middle. Finally, this methodology was used to determine the factors that influence the measurement of noise in a low-cost soundproof booth in chamber to reduce airborne noise in the measurement environment

\section{Keywords:}

Vibration; soundproof cabin; noise level; vehicle; acoustic isolation.

\section{INTRODUCCIÓN}

El objeto de este estudio es analizar los factores que intervienen en la medición de ruido mediante una cabina insonorizada. Esta cabina insonorizada permite simular las condiciones de vibración en el vehículo, de tal forma que las pruebas de detección del ruido se las haga en las instalaciones del productor de la autoparte, sin la necesidad de tener un vehículo, proporcionando el aislamiento acústico adecuado.

Actualmente, el mercado automotriz tiene una alta competitividad, por tanto la calidad del producto es primordial para la satisfacción del cliente. Una característica que aprecia mucho el cliente en un vehículo es la ausencia de ruidos molestos.

Normalmente este tipo de ruidos sólo se los puede detectar una vez que el producto está instalado en el vehículo, con el criterio subjetivo inspector humano, lo cual en la práctica hace difícil la detección de ruidos molestos.

Para realizar la detección de niveles de ruido no conformes en autopartes, se requiere colocar el sistema de medición de ruido como parte del proceso productivo, a manera de una estación de prueba.

Existen varios diseños de cabinas a prueba de ruido, están han sido patentadas. (Ivey, 2018) ha diseñado una cabina recubierta de una lámina de plástico corrugado con un material acústicamente eficaz con líneas de plegado como líneas centrales de ranuras en forma de $V$ plegable para instalación en autos. También se ha propuesto una cabina de insonorización con una estructura con una caja insonorizada que es robusta y ligera, la cual puede absorber el sonido sobre una banda ancha, y ha mejorado el rendimiento de absorción de sonido, el diseño incluye estructura con pequeños hexágonos, tipo panal de abeja (Yamazoe, et al., 2019). La cabina propuesta por (Shiming, et al., 2015) proporciona una mini habitación modular de uso doméstico, en el cual cuerpo de la pared está constituida por una placa acústica de celotex ${ }^{\circledR}$ (fibra de madera aglomerada) y una puerta insonorizada en el cuerpo de la pared.

Para realizar pruebas de test de audición, (Jingyang \& Wanli, 2018) han diseñado una especie de sala insonorizada de prueba auditiva inteligente en la que incluyen los accesorios básicos como una puerta insonorizada controlada automáticamente y una ventana insonorizada, un ventilador y un asiento plegable, para el sistema de inteligencia dispone de una cámara y un intercomunicador, además, una cortina electrónica y un transpondedor que automáticamente realiza la prueba de audición.

En la industria automotriz se ha diseñado por ejemplo una especie de cabina insonorizada adecuada para la prueba del túnel de viento del automóvil, para medir el ruido generado por la escotilla de la ventana de la cabina y el conjunto del parabrisas, esta cabina sirve para validar los niveles de ruido generados por las irregularidades aerodinámicas del vehículo (Hai, 2019).

En el mercado se pueden encontrar cabinas insonorizadas, las cuales sirven para validación, pero no para producción en serie. (iac acoustics, 2019) cámaras insonorizadas de varios tamaños, las cuales por su tamaño no pueden implementarse en una producción en serie de productos electrónicos.

Ninguno de estos diseños es personalizado, de bajo precio y enfocado a una línea de producción.

El sistema de medición de ruido se instalará en el proceso de producción y debe cumplir todos los tiempos del ciclo, a fin de que el tiempo de prueba de niveles de ruido este balanceado con el resto de procesos, debe tener las medidas ergonómicas necesarias para que sea manejado por un inspector, y el sistema debe tener las dimensiones necesarias

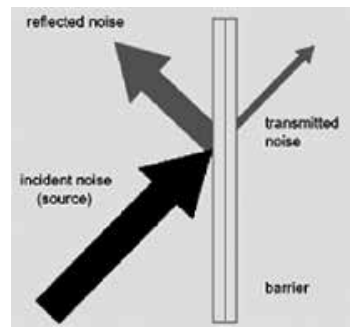

a)

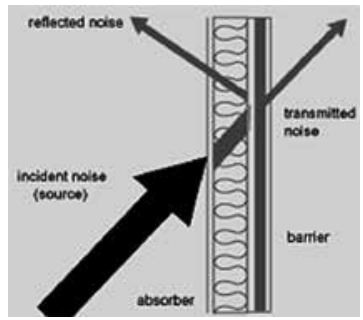

c)

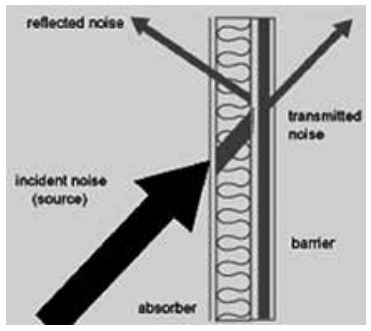

b)

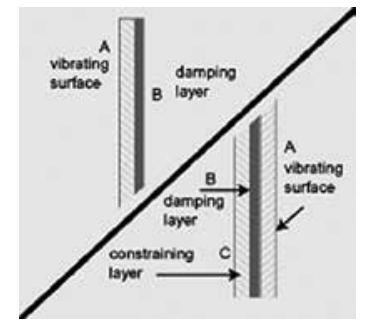

d)

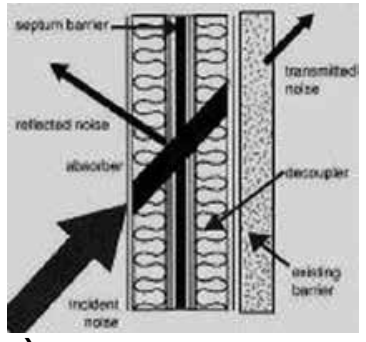

e)

Figura 1 a) Absorbedor b) Barrera c) Composite d) Amortiguador e) Composite desacoplado 
para que ocupe un espacio similar a una estación de prueba, por lo que este sistema no puede colocarse en un sitio apartado del proceso productivo, pues el tiempo de transporte eleva los tiempos de ciclo del proceso.

Dentro de las configuraciones de paredes hacia aislar el ruido externo se pueden encontrar barreras para bloquear la transmisión del sonido, composites que permiten bloquear la transmisión del sonido y reducir las reflexiones de la barrera, absorbedores para reducir la reflexión de sonido y disipar la energía del ruido, amortiguadores para reducir el sonido irradiado de las superficies en vibración y amortiguadores desacoplados que permiten mejorar el rendimiento del material compuesto cuando se aplica al interior de una barrera existente. El desacoplamiento crea un espacio de aire entre la barrera existente y la barrera compuesta del tabique aumentando la pérdida de transmisión más allá de lo que cabría esperar con la conexión directa (Rossing, 2007), ver la Figura 2.

Fu, et al., (2018) realizan el estudio de la pérdida de sonido por transmisión de placas tipo sandwich de estructura de material compuesto laminado sometido a excitación de onda sonora plana, donde las placas de material compuestos laminado se componen de nanotubos de carbono.

El diseño de la cabina sobre el cual se van a analizar los factores que influyen en el nivel de aislamiento se muestran en la Figura 1, dentro de esta cabina se coloca la parte sobre la cual se va a determinar el ruido que genera por vibración. El objetivo es evaluar el nivel de aislamiento acústico de la cabina de tal forma que los ruidos ambientales de una planta de producción no interfieran en la evaluación del nivel de ruido y evaluar sobre esta los factores que influyen en su nivel de aislamiento acústico.

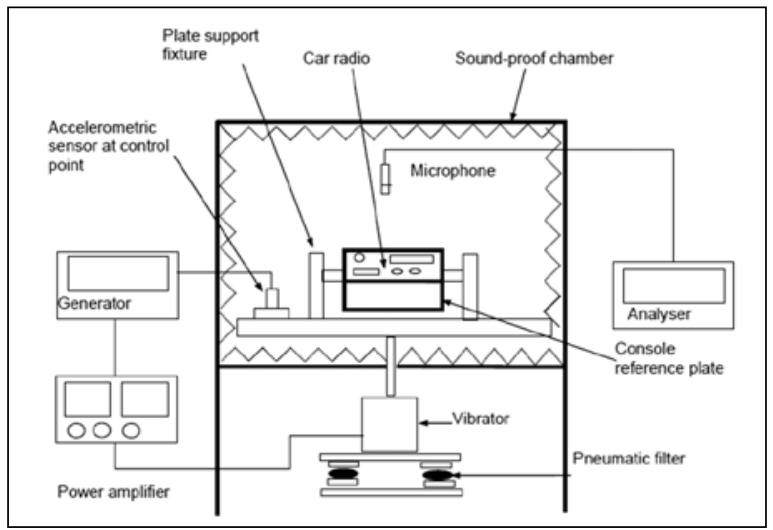

Figura 2. Equipo para generación de vibración y medición de ruido para autopartes electrónicas. Se muestra la cabina (soundproof chamber) como parte del diseño. (Renault, 2004)

El diseño propuesto del sistema de medición, modelado en un software de CAD, montado en una estación de prueba, se muestra en la Figura 2.

La cabina tiene una esponja interna con pirámides o cuñas, las cuales minimizan el efecto de reverberación del sonido (no se muestra en la Figura 2). Esta cabina tiene también las medidas ergonómicas necesarias para un inspector de pie que realice la prueba de 300 unidades al día.

La cabina está configurada por doble pared aislante y un absorbedor de ruido en el medio de las paredes o aire, esta configuración tiene mejores características de aislamiento acústico que una configuración simple (Recuero López, 2000).

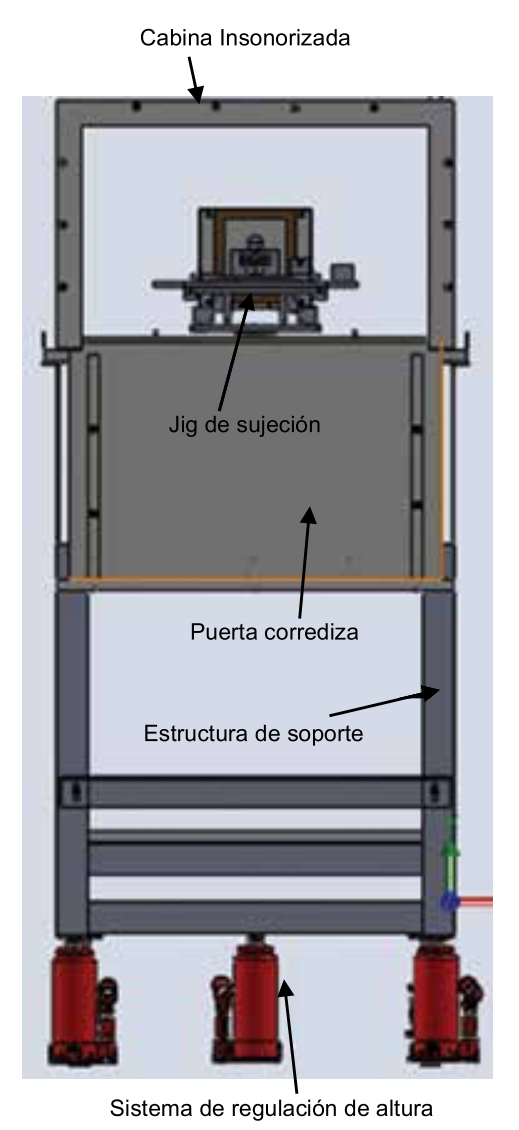

Figura 3. Sistema de medición de ruido de autopartes. Se muestra la cabina y su sistema de soporte y regulación de altura. No se muestra el sistema de generación de vibración. El jig de sujeción se une al sistema de vibración inferior (no se muestra) y sujeta firmemente la parte a prueba. El sistema de regulación permite regular la altura de forma ergonómica. El ancho de la estación de prueba es de $1 \mathrm{~m}$ y su altura máxima de es $180 \mathrm{~cm}$. La puerta corrediza se abre automáticamente para permitir el ingreso de la parte y se cierra herméticamente para evitar el ingreso de ruido.

El diseño y construcción de particiones eficaces es un elemento central en la práctica del control de ruido por parte de ingenieros y arquitectos. Un conocimiento de principios físicos básicos y de buenas prácticas de diseño es importante para un grupo más amplio, incluidos los planificadores de autoridades locales, funcionarios de salud ambiental, funcionarios de edificios y obras, y la gestión industrial. Rao, et al., (2006) estudian los principios básicos mediante análisis de la transmisión de sonido 
a través de algunos modelos idealizados simple de construcciones uniformes de partición de una y dos placas.

Las principales variables para el estudio del aislamiento acústico en una cabina de bajo costo son los materiales, configuraciones de las capas del material, espesor, acople y ruido ambiental del entorno, entre otras que se definen luego.

Con los resultados del estudio se espera diseñar el aislamiento acústico y validar una cabina insonorizada para que proporcione un aislamiento acústico ponderado de mínimo 35dBs, según especifica el requerimiento (Renault, 2004).

Existen diferentes modelos de predicción del índice de reducción acústica. Los principales mecanismos de aumento del aislamiento acústico son el tránsito a través de elementos porosos de ondas acústicas y por cambio de impedancia acústica en la banda de transmisión en el caso de elementos impermeables al sonido.

Los modelos de predicción, en la mayoría de los casos, consideran las frecuencias de resonancia y la frecuencia crítica, las cuales indican las transiciones de aislamiento y los tramos en donde existe posible decaimiento del sonido (Mondaca y González, 2010).

Se puede evaluar los niveles de atenuación acústica a través de análisis numérico y programas de elementos finitos como ANSYS Workbench, por ejemplo, Jie \& Huann, (2017) estudian la pérdida de sonido por transmisión a través de varios tipos de paredes, los resultados de este estudio muestran que se tiene mayor aislamiento acústico configurando paneles o paredes multicapa. Los resultados de este estudio indican que materiales con alta densidad, baja rigidez y alta amortiguación interna son los mejores para construir una barrera al ruido.

Arunkumar, (2018) presenta los estudios realizados para mejorar el comportamiento acústico del panel sandwich de núcleo de celosía, que se utiliza principalmente en aplicaciones estructurales aeroespaciales. El espacio vacío del núcleo de celosía está lleno de espuma de poliuretano (PUF) para lograr mejores características de pérdida de transmisión de sonido. Inicialmente se calculan las propiedades elásticas equivalentes del panel sandwich de núcleo de celosía rellena de espuma. Los resultados revelaron que el llenado con PUF del espacio vacío del núcleo de la viga de celosía reduce significativamente las amplitudes resonantes tanto de las respuestas de vibración como de las acústicas. También se observa que el llenado de espuma reduce significativamente el nivel de potencia sonora general. Del mismo modo, los estudios de pérdida de transmisión sonora revelaron que, las caídas repentinas en las frecuencias de resonancia se reducen significativamente.

\section{Modelos de Predicción del aislamiento acústico}

La ley de masas se usa cuando el aislamiento acústico consta de una sola capa o cuando se tienen varios aislamientos acústicos unidos rígidamente, e indica que las paredes simples se oponen a la energía acústica incidente por medio de su inercia mecánica lo que dificulta la vibración y por tanto la transmisión del sonido, la inercia mecánica está muy ligada al propio peso de la pared.

\section{Algoritmo de Cálculo del nivel de Aislamiento Acústico \\ Índice de Aislamiento Acústico Ponderado}

Para tener un solo número que indique el nivel de aislamiento acústico o pérdida por transmisión, se usa el índice ponderado de reducción sonora Rw para cada configuración de las variables a partir de la curva de aislamiento acústico en cada frecuencia.

Esta pérdida por transmisión, también se representa en una curva en función de la frecuencia, esta curva se la compara con una curva de referencia normalizada, la cual está en bandas de tercios de octava. Esta curva de referencia se muestra en la Figura 4. Para la obtención de Rw se aplica la metodología definida en ISO 717 (ISO, 2013).

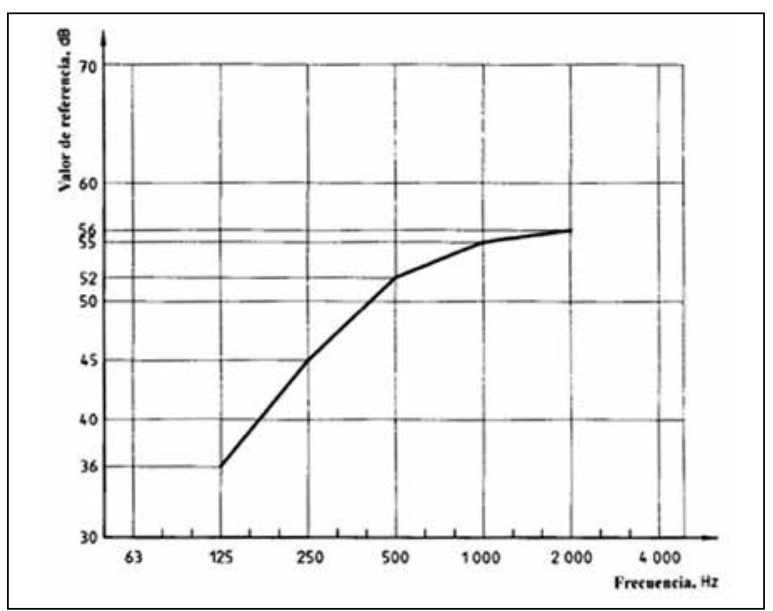

Figura 4. Valores de la curva de referencia para aislamiento a ruido aéreo, en bandas de octava, según ISO 717.

El valor de índice Rw, es el valor correspondiente a la banda de $500 \mathrm{~Hz}$ de la curva de referencia ara aislamiento acústico.

Este número único no toma en consideración la frecuencia, por lo tanto no es muyexacto. Sin embargo, otorga un valor estimado de la configuración de las capas de aislamiento para poder comparar entre ellas.

\section{Ondas de Pliegue}

Las ondas de pliegue son aquellas que se propagan en una placa delgada cuando se la somete a fuerzas perpendiculares al plano de la placa (Lehman Fernández, 2007).

La velocidad de propagación de las ondas de pliegue es:

$$
C_{p}=\sqrt[4]{\frac{\omega^{2} B}{\rho_{s}}},(m / s) \quad \text { EC. }(1)
$$


La rigidez al pliegue en placas sólidas está dada por:

$$
B=\frac{E h^{3}}{12},(N m) \quad \text { Ec. }(2)
$$

$c_{p}=\sqrt{\frac{E}{\rho}} \quad$ Ec. (5)

Donde:

E: módulo de Young $\left[\mathrm{N} / \mathrm{m}^{2}\right]$

h: espesor de la placa [m].

Cuando $c_{p}=c$, ocurre el efecto de coincidencia,

$\sqrt[4]{\frac{\omega^{2} B}{\rho_{s}}}=c \quad(\mathrm{~m} / \mathrm{s}), \quad$ Ec. $(3)$

\section{Aislamiento Acústico para una pared simple}

La pérdida por transmisión de una pared sencilla, $T L$, puede calcularse a través de la ecuación de la masa (Recuero López, 2000), para cada frecuencia, según:

$T L=20 \log \left(\frac{M w}{2 Z}\right)=20 \log f+20 \log M-42 \quad$ Ec. (6)

Donde:

$\omega$ es la frecuencia angular [rad/s]

$\rho_{\mathrm{S}}$ es densidad superficial de la pared $\left[\mathrm{kg} / \mathrm{m}^{2}\right]$

$B$ es rigidez a la flexión [Nm].

$\rho$ es densidad de la pared $\left[\mathrm{kg} / \mathrm{m}^{3}\right]$

$\mathrm{s}$ es el área de la pared [ $\mathrm{m}^{2}$ ]

De esta ecuación se deduce la frecuencia crítica $\mathrm{fc}$, que es la frecuencia a la cual la velocidad de propagación de la onda de pliegue iguala a la velocidad del sonido en el aire (Lehman Fernández, 2007), esta frecuencia está dada por:

$$
f_{c}=\frac{c^{2}}{2 \pi} \sqrt{\frac{\rho_{s}}{B}} \cdot(H z) \quad \text { Ec. }(4)
$$

\section{Dónde:}

c es la velocidad del sonido en el aire.

A esta frecuencia el nivel de aislamiento acústico se reduce debido al fenómeno de resonancia. Cada pared tiene su propia frecuencia crítica. Este fenómeno se muestra en la Figura 5.

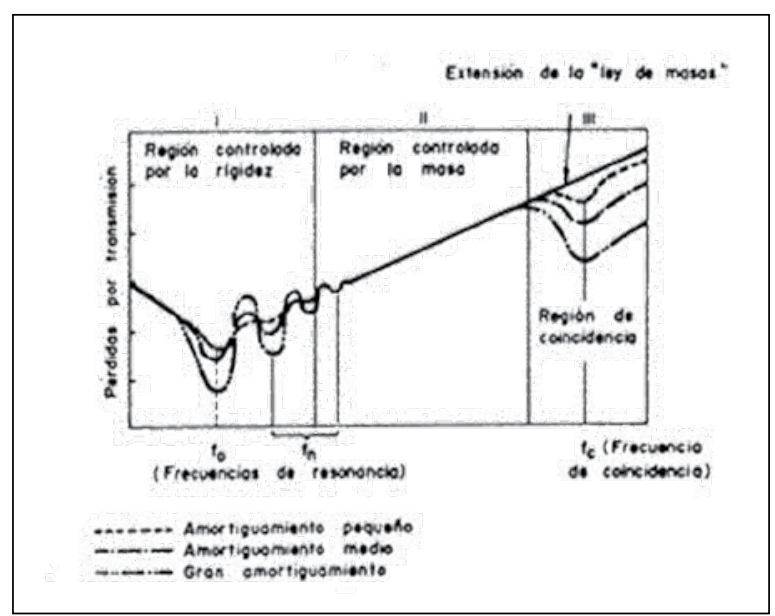

Figura 5. Aislamiento en función de la frecuencia: zona 1 gobernada por la rigidez y la resonancia; zona 2 gobernada por la ley de la masa; zona 3 gobernada por la coincidencia de las ondas. Tomado de (Recuero López, 2000)

Por otro lado, la velocidad del sonido en sólidos está dada por (Thomas D. Rossing, 2007) :

Dónde:

w es la frecuencia angular;

M es la masa por unidad de superficie

Z la impedancia acústica del aire

Este valor de TL indica la pérdida de sonido en dBs entre la fuente y el sitio de medición.

En la ecuación 6, se considera que la onda incide de forma perpendicular a la pared, en el caso de incidencia aleatoria se cambia el valor de 42 por 47.

\section{Aislamiento acústico para paredes dobles}

Existen diversos métodos para el aislamiento acústico con paredes dobles, entre los cuales se pueden mencionar:

- Ley de masa de una pared doble a incidencia normal

- Ley de masa a Incidencia aleatoria

- Ley de Masa a Incidencia Normal intuitiva

- Método aproximado de Robert Jones

- Corrección de Sharp a la ley de Masa incidencia aleatoria

- Método práctico de Iwashige \& Ohta

- Aproximación de Brekke al Método SEA

- Método de Impedancia Progresiva de Fringuellino \& Guglielmone

Se ha escogido para este estudio el método de Sharp que da los resultados más exactos. Este método es una corrección a ley de la masa de incidencia aleatoria (Lehman Fernández, 2007).

Un sistema de aislamiento acústico de pared doble consiste en dos paredes simples separadas por un material elástico o aire.

El nivel de aislamiento acústico depende de las características acústicas de las paredes, el tipo de acoplamiento entre ellas, y la absorción acústica del componente en la cavidad entre las dos paredes. Las dos posibles configuraciones para la cabina de este estudio se muestran en la Figura 4 y en la Figura 5.

La ley de las masas, que se usa típicamente para el cálculo del aislamiento acústico, divide el panel doble en tres regiones, bajo la frecuencia de resonancia, entre la frecuencia de resonancia y la frecuencia de transición y sobre la frecuencia de transición (Thomas D. Rossing, 2007).

En el modelo corregido de Sharp, la pérdida 
de transmisión del sistema de pared doble ya no está determinada por 2 frecuencias, sino por 5 frecuencias: la resonancia menor del sistema, la frecuencia de coincidencia de cada uno de los paneles, la resonancia más baja del sistema acústico, una frecuencia de puente ( $\mathrm{fp}$, calculada a partir de las 2 rectas interceptadas por ella) y la frecuencia límite, que tiene que ver con la separación entre paneles (Lehman, 2007).

La resonancia de la cavidad $f_{0}$ está dada por:

$$
f_{0}=\frac{1}{2 \pi}\left(\frac{1.8 \gamma P \cdot\left(m_{1}+m_{2}\right)}{d \cdot m_{1} \cdot m_{2}}\right)^{\frac{1}{2}}(H z) \quad \text { Ec. (7) }
$$

Donde:

$\mathrm{m} 1, \mathrm{~m} 2$ son las densidades superficiales de los paneles 1 y 2 , respectivamente $(\mathrm{kg} / \mathrm{m} 2)$

$\mathrm{d}$, la distancia entre los paneles $(\mathrm{m})$

$g$, es la relación de calores específicos del aire, $\mathrm{y}:=1.41$

P es la presión estática del aire, $P=101.3 \mathrm{kPa}$

La frecuencia límite, $f_{l}$, que está relacionada con el espesor de la cavidad, se calcula:

$$
f_{l}=\frac{c}{2 \cdot \pi \cdot d}(\mathrm{~Hz})
$$

Las frecuencias críticas $f_{c}$ de cada panel están definidas por:

$$
f_{c}=\frac{c^{2}}{2 \pi} \sqrt{\frac{\rho_{s}}{B}} \cdot(H z)
$$

Dónde:

$\rho_{\mathrm{S}}$ : densidad superficial del sólido $\left[\mathrm{kg} / \mathrm{m}^{2}\right]$

B: rigidez a la flexión del sólido, [ $\mathrm{Nm}]$.

c: velocidad del sonido en el aire.

Esta frecuencia crítica es la frecuencia a la cual la velocidad de propagación de la onda de pliegue iguala a la velocidad del sonido en el aire.

$f_{p}$ es la frecuencia de puente y está relacionada con la frecuencia a la cual se tienen un aumento en la pérdida por transmisión debida a la unión estructural de los paneles con una viga, tal como se indica en la Figura 8.

En resumen, para este método existen frecuencias de importancia f0, fp, fC1 y $0.5 f C 2$, que definen 5 regiones, con cada una de estas frecuencias se forma el par ordenado (fi, TLi), para el cálculo de la pérdida por transmisión.

La pérdida por transmisión $T L$ en $d B$ para la configuración de paredes dobles está dada por:

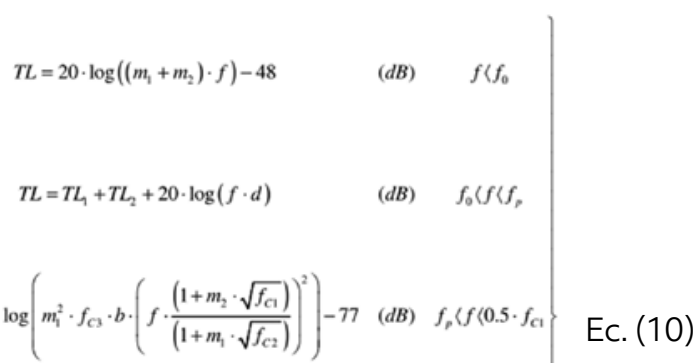

$$
\begin{aligned}
& \left.T L=\log \left(\frac{f}{\left(0.5 \cdot f_{C 1}\right)}\right) \cdot\left(\frac{T L_{C}-T L_{s}}{\log \left(f_{C S} /\left(0.5 \cdot f_{C 1}\right)\right.}\right)\right)+T L_{B} \quad(d B) \quad 0.5 \cdot f_{C 1}\left\langle f_{C 2}\right. \\
& T L=50 \cdot \log \left(\frac{10^{\frac{m_{c}}{s^{5}}}}{f_{C 2}} \cdot f\right) \quad(d B) \quad f>f_{C 2}
\end{aligned}
$$

Dónde:

TL es la perdida por transmisión

$\mathrm{TL}_{1}$ es la pérdida por transmisión en la pared 1

$\mathrm{TL}_{2}$ es la pérdida por transmisión en la pared 2 fp es la frecuencia de puente

De (Sharp, 1973) se tiene que:

$T L_{B 1}=T L_{A}+20 \cdot \log \left(f_{C 1} / f_{0}\right)-6(d B)$

Dónde:

$T L_{A}=20 \cdot \log \left(\left(m_{1}+m_{2}\right) f_{0}\right)-48(d B) ;$

b es el espaciamiento entre vigas

$\mathrm{f}_{\mathrm{c} 1}$ es la frecuencia crítica de la pared 1

$f_{c 2}$ es la frecuencia crítica de la pared 2

TLC corresponde a la pérdida por transmisión para $0.5 f_{\mathrm{C} 1}$

TLB corresponde a la pérdida por transmisión para $\mathrm{f}_{\mathrm{C} 2}$

$T L_{B 2}=10 \cdot \log \left(\left(m_{1}^{2} \cdot b \cdot f_{C 2}^{3}\right) \cdot\left(1+\frac{m_{2} \sqrt{f_{C 1}}}{m_{1} \sqrt{f_{C 2}}}\right)^{2}\right)-77(d B) \quad$ EC. $(13)$
TL $L_{B}$ corresponde al mayor entre $\mathrm{TL}_{\mathrm{B} 1 \mathrm{Y}} \mathrm{TL}_{\mathrm{B} 2}$
Si las paredes son iguales $\mathrm{fc} 1=\mathrm{fc} 2$, de donde:

$T L_{C}=T L_{B}+6+10 \cdot \log \left(\eta_{2}\right)+5 \cdot \log \left(\eta_{1}\right) \quad(d B) \quad$ Ec. (14)

Si las paredes son diferentes:

$T L_{C}=T L_{B}+6+10 \cdot \log \left(\eta_{2}\right)$

Dónde $\eta_{1}, 2$ son los factores de pérdidas del material de la pared 1 y 2 , respectivamente. 


\section{Propiedades de los materiales:}

En la tabla 1 se muestran los valores de las propiedades físicas y mecánicas de los materiales aislantes usadas en este estudio.

Tabla 1. Propiedades mecánicas y físicas de los materiales para los cálculos de los índices de aislamiento acústico

\begin{tabular}{|l|l|l|l|}
\hline \multicolumn{1}{|c|}{ Propiedad } & \multicolumn{1}{|c|}{ Unidades } & \multicolumn{1}{|c|}{ MDF } & \multicolumn{1}{|c|}{ OSB } \\
\hline $\begin{array}{l}\text { Módulo de } \\
\text { Young E }\end{array}$ & {$[\mathrm{Gpa}]$} & 2.5 & 2.55 \\
\hline $\begin{array}{l}\text { Densidad } \mathrm{r} \\
{[\mathrm{kg} / \mathrm{m} 3]}\end{array}$ & 616.51 & 691.03 \\
\hline $\begin{array}{l}\text { Factor de } \\
\text { Perdida } \mathrm{h}\end{array}$ & - & 0.038 & 0.031 \\
\hline $\begin{array}{l}\text { Coeficiente de } \\
\text { Poisson } \mathrm{m}\end{array}$ & - & 0.25 & 0.25 \\
\hline $\begin{array}{l}\text { Velocidad de } \\
\text { propagación } \\
\text { longitudinal } \\
\text { del sonido }\end{array}$ & $\mathrm{m} / \mathrm{s}$ & 2098.25 & 2098.25 \\
\hline
\end{tabular}

El coeficiente de absorción acústica medio aw, se lo calcula mediante el método usado en ISO 11654. Para este caso se usa el valor de 0.5 para poliuretano de $40 \mathrm{~kg} / \mathrm{m} 3$ de densidad (ATEPA, 2016).

Un método simplificado para el cálculo del aislamiento acústico (TL) se da en las ecuación 15,16 y 17, dependiendo de la frecuencia (Jones, 1981):

$$
R=20 \log \left(f\left(m_{1}+m_{2}\right)\right)-47 f<f_{O}
$$

$R=R_{1}+R_{2}+20 \log (f d)-29 f_{o}<f<f_{l}$

$\mathrm{R}=R_{1}+\mathrm{R}_{2}+6$

\section{MATERIALES Y MÉTODOS}

Esta es una investigación aplicada, ya que se usará la teoría de aislamiento acústico y se la aplicará al caso concreto de una cabina insonorizada con aislamiento tipo sandwich previamente diseñada.

Se realizó la búsqueda bibliográfica en la base de datos Science Direct, el objetivo principal de la revisión fue encontrar metodologías de la evaluación del aislamiento acústico de paneles dobles con absorbente en el medio, porque este diseño tiene la cabina en estudio. Se seleccionaron los métodos de cálculo con más citas, que acercan más los resultados del estudio a mediciones reales, y métodos que no impliquen gran complejidad matemática.

\section{Definición de factores de aislamiento acústico}

Se definieron, mediante el uso de un diagrama de causa - efecto y lluvia de ideas, los principales factores que podrían afectar a la medición del nivel de ruido y el aislamiento acústico de la cabina son:

- Número de paredes

- Número de capas de cada pared

- Espesor de cada pared*
- Material del aislante de cada pared*

- Separación entre paredes*

- Material del absorbente*

- Espesor del material del absorbente*

- Tipo de acople entre paredes*

- Dimensiones de las paredes*

De estos factores que afectan la medición del ruido, se clasificaron en factores controlables y factores no controlables, se escogieron 7 factores, los cuales en el listado anterior se marcan con asterisco, y se consideran que más influyen en los resultados de la medición. Estos factores son:

- Espesor del aislante de cabina insonorizada, los espesores corresponden a materiales estándar encontrados en el mercado ecuatoriano.

- Coeficiente de absorción sonora del material, en este caso se usan los valores del MDF (Medium Density Fiber Board) y OSB (Oriented Strand Board). Ver la Tabla 1.

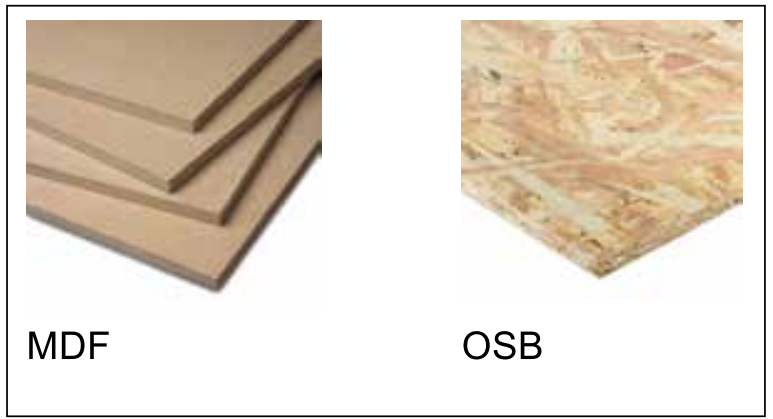

Figura 6. Tableros de bajo costo usados para el análisis del aislamiento acústico

- Área superficial de la cabina, se realiza el análisis en dos cabinas de medidas ergonómicas para realización de inspección en serie. El volumen de la cabina permite conocer el largo y ancho, el cual influye en el nivel de aislamiento (Recuero López, 2000).

- Configuración de capas de material aislante, se usan la configuración tipo sánduche con variantes de una barrera acústica y aire en el medio. La barrera acústica evita la transmisión de ruido aéreo a través de la estructura de la cabina, tal como se muestra en la Figura 7.

La barrera o absorbente acústico usado para el estudio es de poliuretano de $40 \mathrm{~kg} / \mathrm{m}^{3}$ de densidad.

- La separación entre paredes está dada por el espesor de absorbente acústico, se realizó el análisis con el poliuretano y con aire en el medio. Esta distancia d se muestra en la Figura 8.

- El acople o unión entre las paredes es importante ya que esta unión permite la transmisión de la vibración ocasionada por las ondas de sonido. Se usó un acople con madera separada a la altura del tablero y otra configuración sin acople como se muestra en la Figura 8. 


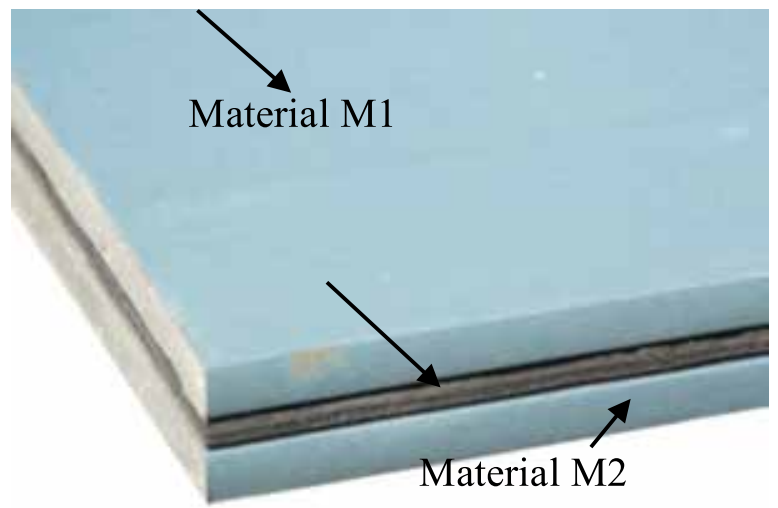

Figura 7. Configuración tipo sánduche para el análisis del aislamiento acústico. Adaptado de (Systems, 2017)

Los factores no controlables, a los cuales se fijó en el cálculo, son los siguientes:

- Punto de ubicación de micrófono, de modo que no afecte la medición.

- Sujeción de cabina a estructura, para que no transmita vibración a la cabina.

- Sensibilidad del micrófono, para que detecte los mínimos cambios de niveles de ruido.

- Nivel de reverberación.

- Número de capas de cada pared se mantendrá en una.

- Se usará un análisis con paredes dobles.
Se usó un diseño factorial $2 \mathrm{~K}$, para lo cual se definieron los niveles de los factores mostrados en la Tabla 2.

Se utilizó el software Minitab versión 17 para crear un diseño cribado de 7 factores, con el fin de facilitar la combinación de estos. En la Tabla 3. Se muestra la combinación de factores para determinar el nivel de aislamiento.

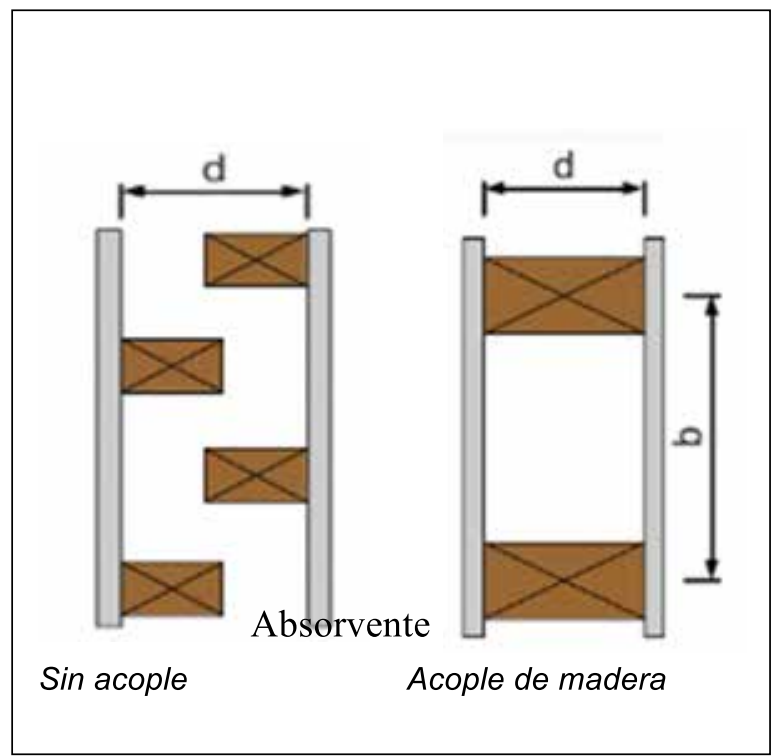

Figura 8. Configuración de las paredes de la cabina. d es la distancia entre las paredes, $b$ es la distancia entre acoples.

Tabla 2. Niveles de Variables Controlables en diseño factorial $2 \mathrm{~K}$

\begin{tabular}{|l|l|l|}
\hline \multicolumn{1}{|c|}{ Factor } & \multicolumn{1}{|c|}{ Nivel Bajo } & \multicolumn{1}{c|}{ Nivel Alto } \\
\hline Espesor de pared aislante 1 $[\mathrm{mm}]$ & 20 & 30 \\
\hline Espesor pared aislante 2 $[\mathrm{cm}]$ & 20 & 30 \\
\hline Material Aislante pared 1 & MDF & OSB \\
\hline Material Aislante de la pared 2 & MDF & OSB \\
\hline Separación entre paredes* [mm] & 10 & 20 \\
\hline Tipo de acople & Sin acople & Madera \\
\hline Alto y ancho de la pared & $60 \mathrm{~cm} \times 60 \mathrm{~cm}$ & $80 \mathrm{~cm} \times 80 \mathrm{~cm}$ \\
\hline
\end{tabular}

${ }^{*}$ El espesor del absorbente coincide con la separación de las paredes

Tabla 3. Combinación de factores para determinar el nivel de aislamiento acústico

\begin{tabular}{|c|c|c|c|c|c|c|c|c|}
\hline Trat. & $\begin{array}{c}\text { Espesor } \\
\text { pared 1 } \\
(\mathbf{m m})\end{array}$ & $\begin{array}{c}\text { Espesor } \\
\text { pared 2 } \\
(\mathbf{m m})\end{array}$ & $\begin{array}{c}\text { Separación } \\
\text { Entre paredes } \\
(\mathbf{m m})\end{array}$ & Material 1 & Material 2 & $\begin{array}{c}\text { Alto-Ancho } \\
(\mathbf{m m \times m m})\end{array}$ & Acople & Absorbente \\
\hline 1 & 20 & 30 & 20 & MDF & OSB & $60 \times 60$ & Sin acople & Poliuretano \\
\hline 2 & 20 & 30 & 20 & OSB & MDF & $80 \times 80$ & Madera & Poliuretano \\
\hline 3 & 20 & 30 & 10 & MDF & MDF & $80 \times 80$ & Madera & Aire \\
\hline 4 & 30 & 30 & 10 & OSB & MDF & $60 \times 60$ & Sin acople & Aire \\
\hline 5 & 30 & 20 & 20 & OSB & MDF & $80 \times 80$ & Sin acople & Poliuretano \\
\hline 6 & 20 & 20 & 20 & OSB & OSB & $60 \times 60$ & Madera & Aire \\
\hline 7 & 20 & 20 & 10 & MDF & MDF & $60 \times 60$ & Sin acople & Poliuretano \\
\hline 8 & 30 & 30 & 10 & OSB & OSB & $60 \times 60$ & Madera & Poliuretano \\
\hline 9 & 20 & 20 & 10 & OSB & OSB & $80 \times 80$ & Sin acople & Aire \\
\hline 10 & 30 & 30 & 20 & MDF & OSB & $80 \times 80$ & Sin acople & Aire \\
\hline 11 & 30 & 20 & 20 & MDF & MDF & $60 \times 60$ & Madera & Aire \\
\hline 12 & 30 & 20 & 10 & MDF & OSB & $80 \times 80$ & Madera & Poliuretano \\
\hline
\end{tabular}


Con los niveles definidos de cada factor, se ingresaron los valores de estas variables en la hoja de cálculo, para definir el nivel de aislamiento acústico, aplicando el algoritmo de cálculo del nivel de aislamiento acústico del método Sharp.

La distancia b, referente a la distancia entre acoples se igualó al valor de la altura de la pared en cada caso.

\section{MÉTODO PARA ANÁLISIS DE RESULTADOS}

Finalmente, para validar los resultados se compararon los resultados de la simulación con los cálculos aplicando el método de Sharp y Jones y se evalúo el error entre los dos métodos. Se realizaron

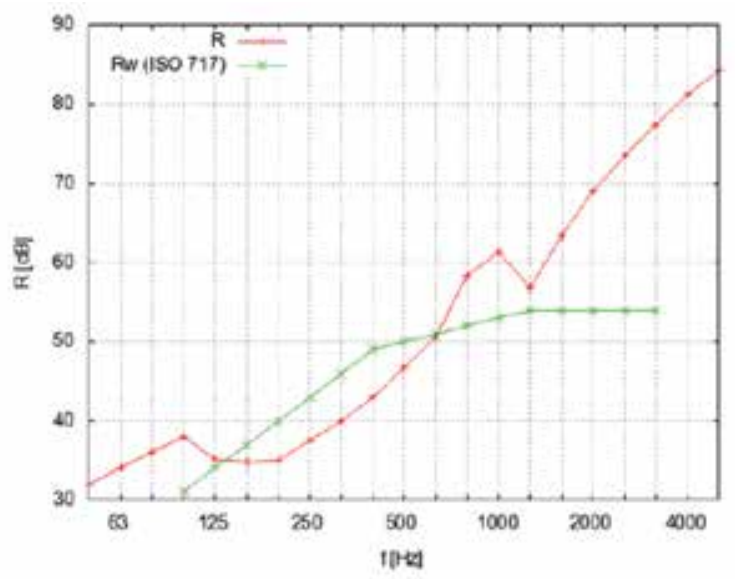

Figura 9. Resultados del índice de aislamiento acústico para el rango de frecuencia de $0-5000 \mathrm{~Hz}$ para el tratamiento 1 . La curva verde representa el aislamiento acústico según ISO 177.

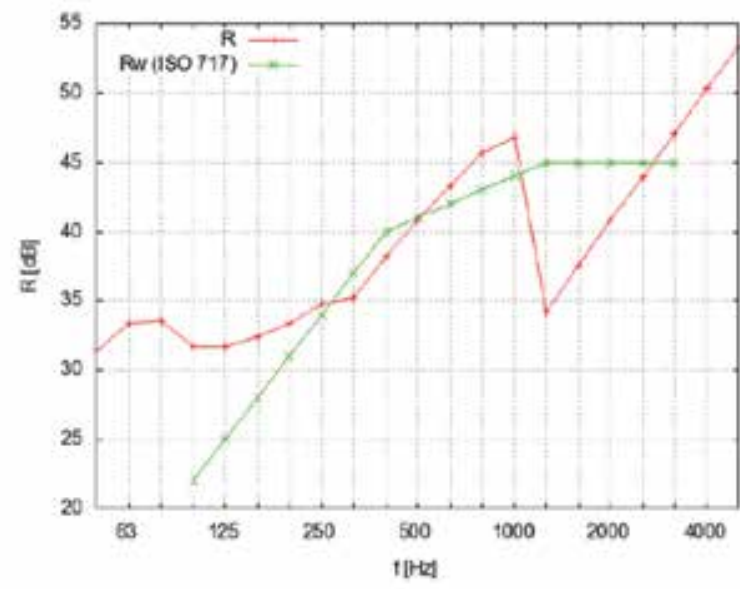

Figura 10. Resultados del índice de aislamiento acústico para el rango de frecuencia de $0-5000 \mathrm{~Hz}$ para el tratamiento 2 . gráficos del aislamiento en función de la frecuencia incidente para la simulación con el programa dBwave y los cálculos con los métodos revisados en la bibliografía.

\section{RESULTADOS}

Los resultados de cada combinación de factores, luego de aplicar la ecuación 16 se ingresaron en la columna "Índice de Aislamiento Acústico Ponderado Rw" .

Se muestran los resultados del índice de aislamiento acústico para los 12 tratamientos o combinaciones, desde la Figura 9 hasta la Figura 20, para distintas frecuencias, en bandas de tercio de octava. En cada una de las figuras se muestra en verde la curva de referencia según ISO 717.

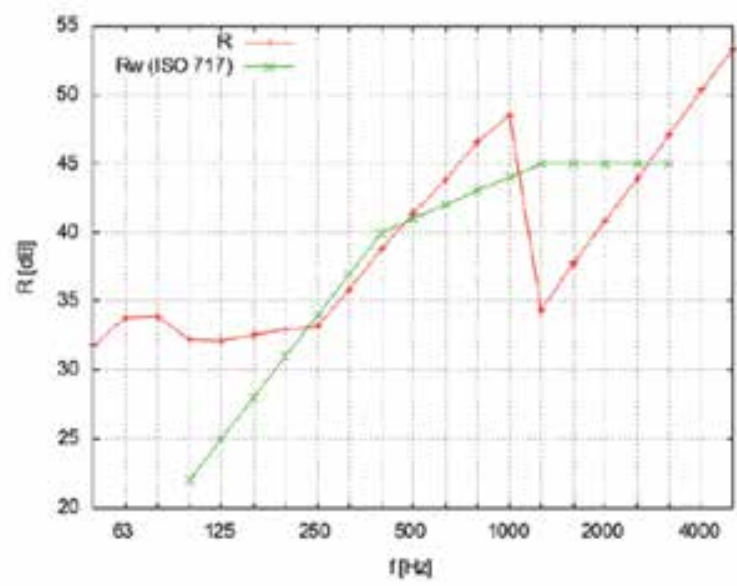

Figura 11. Resultados del índice de aislamiento acústico para el rango de frecuencia de $0-5000 \mathrm{~Hz}$ para el tratamiento 3.

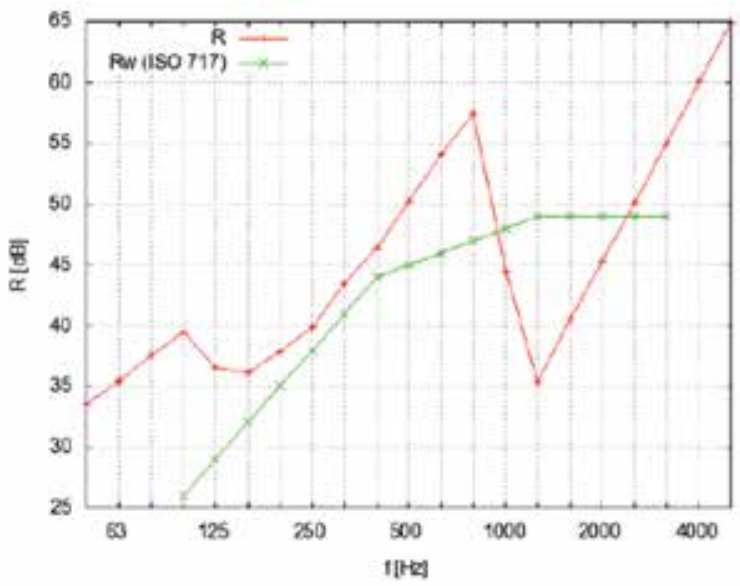

Figura 12. Resultados del índice de aislamiento acústico para el rango de frecuencia de $0-5000 \mathrm{~Hz}$ para el tratamiento 4. 


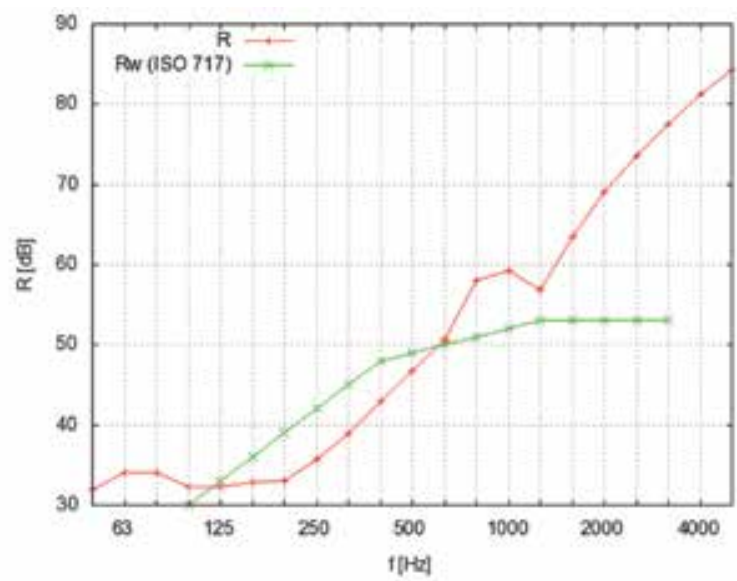

Figura 13. Resultados del índice de aislamiento acústico para el rango de frecuencia de $0-5000 \mathrm{~Hz}$ para el tratamiento 5 .

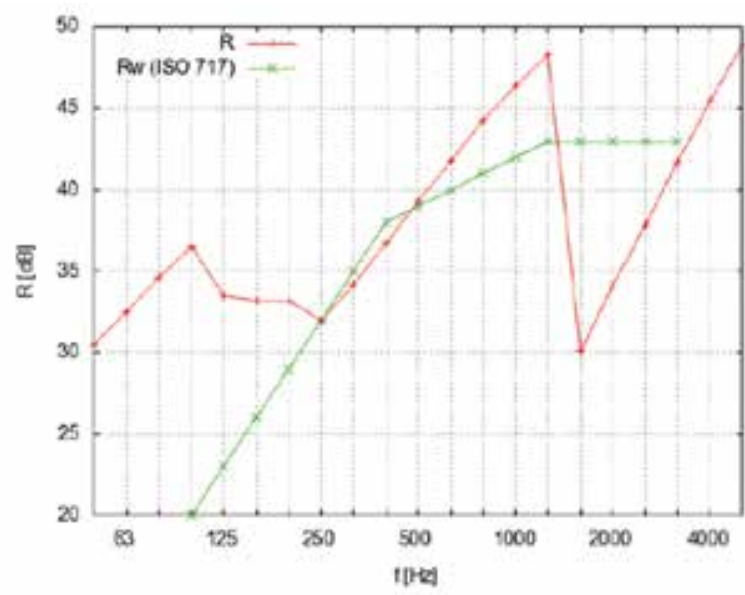

Figura 14. Resultados del índice de aislamiento acústico para el rango de frecuencia de $0-5000 \mathrm{~Hz}$ para el tratamiento 6.

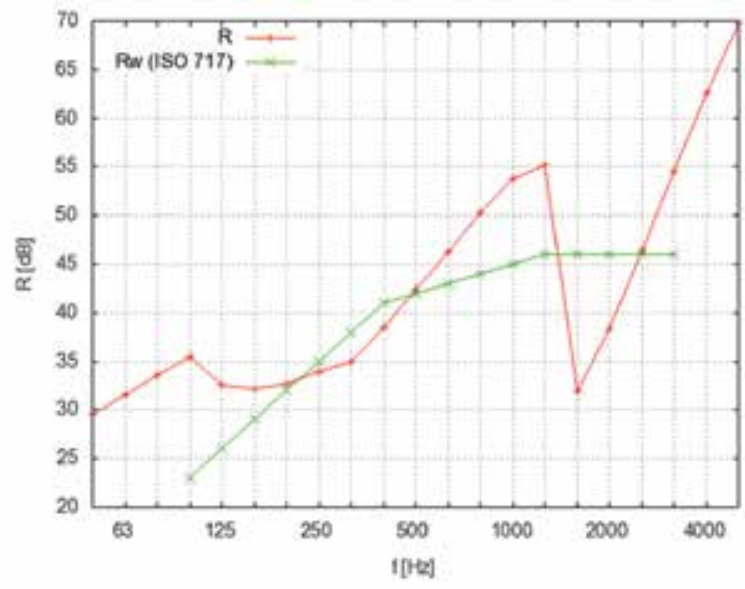

Figura 15. Resultados del índice de aislamiento acústico para el rango de frecuencia de $0-5000 \mathrm{~Hz}$ para el tratamiento 7 .

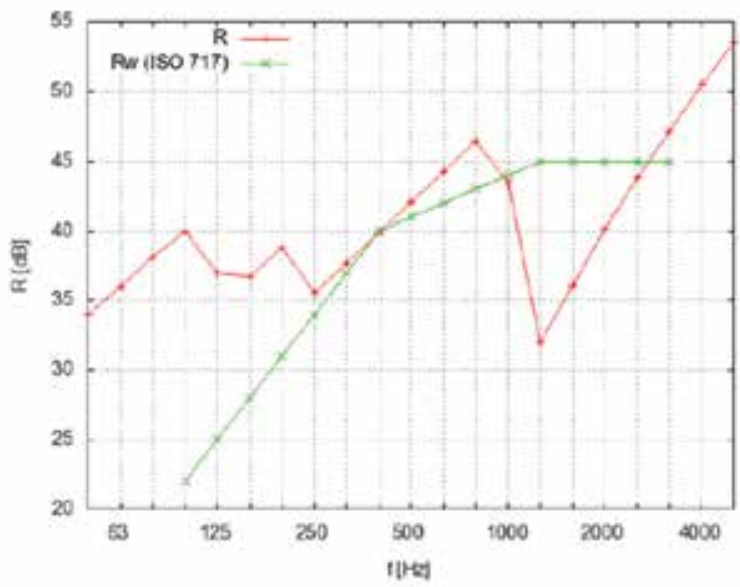

Figura 16. Resultados del índice de aislamiento acústico para el rango de frecuencia de $0-5000 \mathrm{~Hz}$ para el tratamiento 8 .

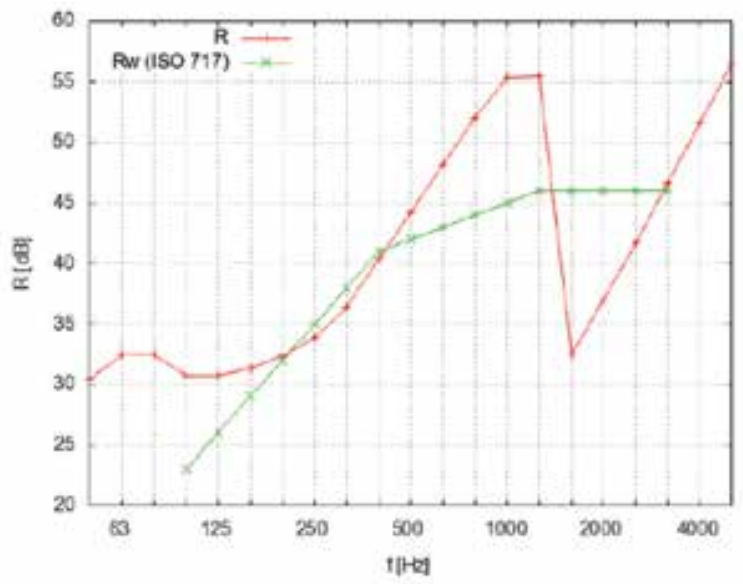

Figura 17. Resultados del índice de aislamiento acústico para el rango de frecuencia de $0-5000 \mathrm{~Hz}$ para el tratamiento 9 .

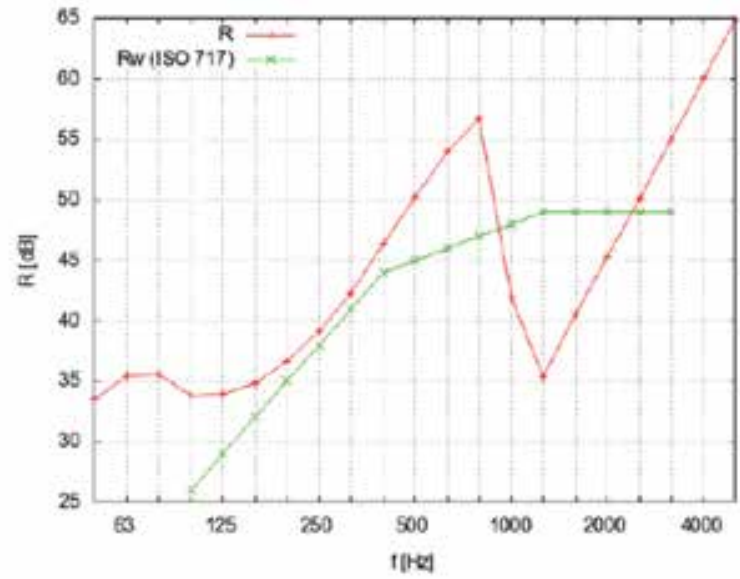

Figura 18. Resultados del índice de aislamiento acústico para el rango de frecuencia de $0-5000 \mathrm{~Hz}$ para el tratamiento 10 . 


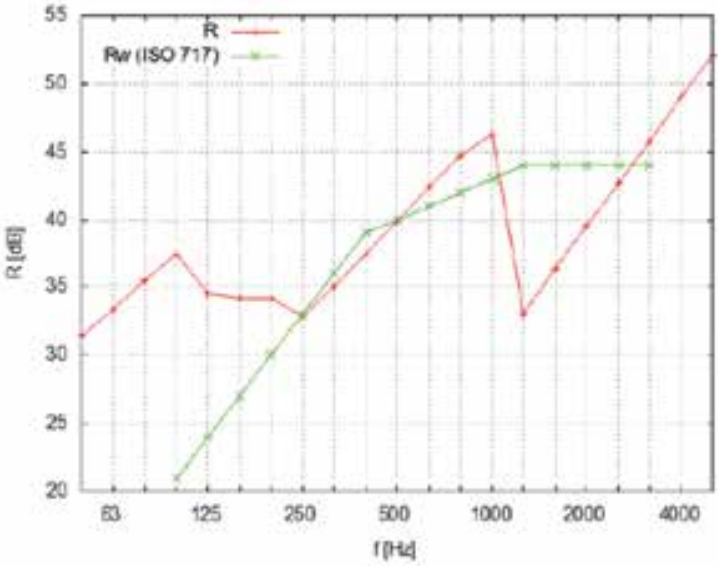

Figura 19. Resultados del índice de aislamiento acústico para el rango de frecuencia de $0-5000 \mathrm{~Hz}$ para el tratamiento 11 .

En la Tabla 4, se muestran el índice de aislamiento acústico LT para cada tratamiento y para cada

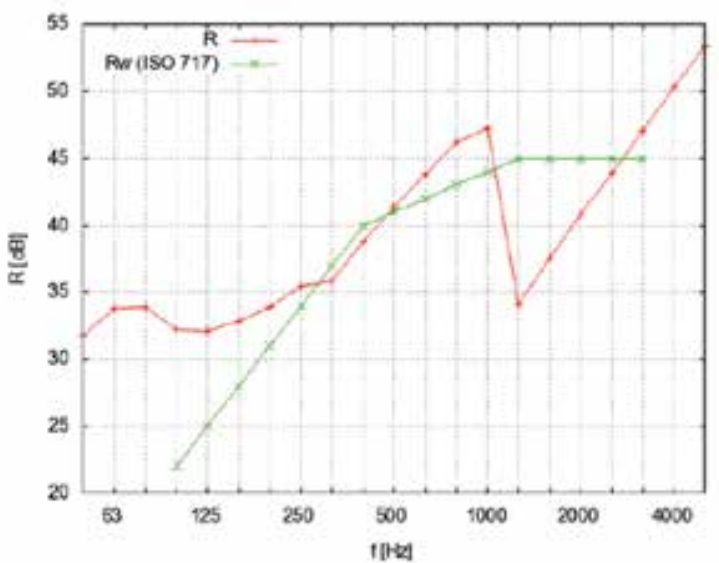

Figura 20. Resultados del índice de aislamiento acústico para el rango de frecuencia de $0-5000 \mathrm{~Hz}$ para el tratamiento 12 .

frecuencia en bandas de tercio de octava para cada combinación de variables o tratamientos.

Tabla 4. Resultado del cálculo del aislamiento acústico para cada tratamiento o combinación de factores con el método de Sharp para cada frecuencia

\begin{tabular}{|c|c|c|c|c|c|c|c|c|c|c|c|c|}
\hline \multicolumn{13}{|c|}{ Tratamiento } \\
\hline $\begin{array}{c}\text { Frecuencia } \\
{[\mathrm{Hz}]}\end{array}$ & 1 & 2 & 3 & 4 & 5 & 6 & 7 & 8 & 9 & 10 & 11 & 12 \\
\hline 50 & 32.0 & 31.8 & 31.4 & 33.5 & 32.0 & 30.5 & 29.5 & 34.0 & 30.5 & 33.5 & 31.4 & 31.8 \\
\hline 63 & 34.0 & 33.8 & 33.4 & 35.5 & 34.0 & 32.5 & 31.5 & 36.0 & 32.5 & 35.5 & 33.4 & 33.8 \\
\hline 80 & 36.1 & 33.9 & 33.5 & 37.6 & 34.1 & 34.6 & 33.6 & 38.1 & 32.5 & 35.6 & 35.5 & 33.9 \\
\hline 100 & 38.0 & 32.2 & 31.7 & 39.5 & 32.3 & 36.5 & 35.5 & 40.0 & 30.8 & 33.8 & 37.4 & 32.2 \\
\hline 125 & 35.1 & 32.1 & 31.7 & 36.6 & 32.3 & 33.5 & 32.5 & 37.0 & 30.8 & 33.9 & 34.5 & 32.1 \\
\hline 160 & 34.8 & 32.6 & 32.4 & 36.2 & 32.8 & 33.2 & 32.2 & 36.7 & 31.4 & 34.8 & 34.2 & 32.8 \\
\hline 200 & 35.0 & 32.9 & 33.4 & 37.9 & 33.0 & 33.2 & 32.7 & 38.8 & 32.4 & 36.7 & 34.2 & 33.9 \\
\hline 250 & 37.5 & 33.2 & 34.8 & 39.9 & 35.7 & 32.0 & 33.9 & 35.6 & 33.9 & 39.2 & 32.8 & 35.5 \\
\hline 315 & 40.0 & 35.8 & 35.2 & 43.5 & 38.9 & 34.2 & 35.0 & 37.7 & 36.4 & 42.3 & 35.0 & 35.8 \\
\hline 400 & 42.9 & 38.8 & 38.2 & 46.4 & 42.9 & 36.7 & 38.5 & 39.9 & 40.4 & 46.4 & 37.4 & 38.8 \\
\hline 500 & 46.8 & 41.4 & 40.8 & 50.2 & 46.7 & 39.3 & 42.3 & 42.1 & 44.2 & 50.2 & 39.9 & 41.4 \\
\hline 630 & 50.7 & 43.8 & 43.3 & 54.1 & 50.6 & 41.8 & 46.2 & 44.3 & 48.2 & 54.0 & 42.4 & 43.8 \\
\hline 800 & 58.3 & 46.6 & 45.7 & 57.5 & 58.0 & 44.3 & 50.3 & 46.5 & 52.1 & 56.8 & 44.7 & 46.2 \\
\hline 1000 & 61.3 & 48.5 & 46.8 & 44.5 & 59.3 & 46.4 & 53.8 & 43.5 & 55.4 & 41.9 & 46.3 & 47.3 \\
\hline 1250 & 56.8 & 34.3 & 34.2 & 35.4 & 56.8 & 48.3 & 55.2 & 32.0 & 55.5 & 35.4 & 33.0 & 34.1 \\
\hline 1600 & 63.5 & 37.8 & 37.6 & 40.6 & 63.5 & 30.1 & 31.9 & 36.1 & 32.5 & 40.6 & 36.4 & 37.6 \\
\hline 2000 & 69.0 & 40.8 & 40.8 & 45.3 & 69.0 & 34.1 & 38.3 & 40.1 & 37.0 & 45.3 & 39.5 & 40.8 \\
\hline 2500 & 73.5 & 43.9 & 43.9 & 50.1 & 73.5 & 37.9 & 46.4 & 43.8 & 41.7 & 50.1 & 42.7 & 43.9 \\
\hline 3150 & 77.5 & 47.1 & 47.1 & 55.0 & 77.5 & 41.7 & 54.5 & 47.2 & 46.6 & 55.0 & 45.8 & 47.1 \\
\hline 4000 & 81.2 & 50.3 & 50.3 & 60.1 & 81.2 & 45.5 & 62.6 & 50.5 & 51.7 & 60.1 & 49.1 & 50.3 \\
\hline 5000 & 84.3 & 53.3 & 53.3 & 64.9 & 84.3 & 48.9 & 69.7 & 53.5 & 56.5 & 64.9 & 52.1 & 53.3 \\
\hline
\end{tabular}


En la Tabla 5 se muestra el índice de aislamiento acústico ponderado para cada tratamiento.

\begin{tabular}{|c|c|}
\hline Tratamiento & $\begin{array}{c}\text { Índice de Aislamiento } \\
\text { Acústico Ponderado Rw (dB) }\end{array}$ \\
\hline 1 & 50 \\
\hline 2 & 41 \\
\hline 3 & 41 \\
\hline 4 & 45 \\
\hline 5 & 49 \\
\hline 6 & 39 \\
\hline 7 & 42 \\
\hline 8 & 41 \\
\hline 10 & 42 \\
\hline 11 & 45 \\
\hline 12 & 40 \\
\hline
\end{tabular}

Tabla 5. Resultado del índice de aislamiento acústico ponderado para cada tratamiento.

La Figura 21, muestra el análisis de Pareto de las variables que influyen en el aislamiento acústico, a partir del diseño factorial 2K.

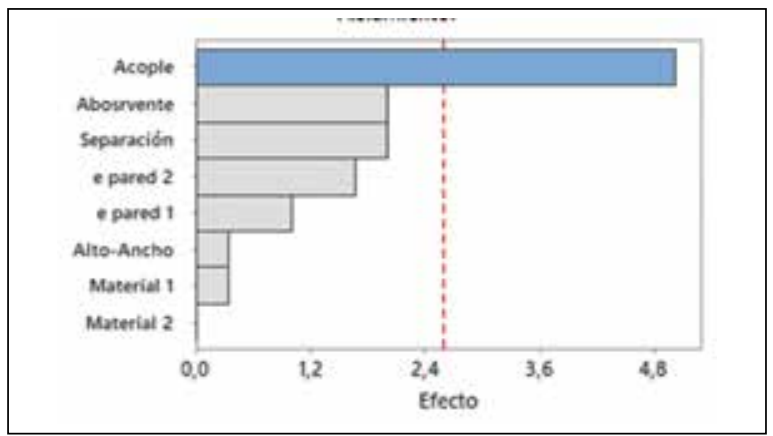

Figura 21. Diagrama de Pareto de efectos. Los factores con barras más largas tienen más influencia sobre el aislamiento, en este caso el acople entre paneles. La línea punteada es el tamaño del efecto en el nivel de significancia 0,10. Las barras grises representan términos no significativos que podrían ser eliminados del modelo.
La Figura 21, indica como cada factor o variable influye en la respuesta, es decir en el nivel de aislamiento acústico ponderado.

En la figura 23, se hace una comparación de los niveles de aislamiento obtenidos con las dos 12 combinaciones de material, espesor y aislamiento, se puede observar que el mayor nivel de aislamiento acústico se obtienen con la combinación 5 , en la cual se tienen un panel de $30 \mathrm{~mm}$ de OSB, $20 \mathrm{~mm}$ de MDF, un espacio de $20 \mathrm{~mm}$, sin acople con absorbente de poliuretano.

\section{DISCUSIÓN DE RESULTADOS}

Tal como lo indican varios autores (Sharp, 1973), (Lehman Fernández, 2007) (Rao, et al., 2006) el mayor aislamiento se tiene cuando existe una variación de impedancias acústicas entre paneles, esto se obtiene cuando los materiales de los paneles son diferentes, se tiene una mayor separación entre paneles y en la cavidad resultante se pone un material con gran absorción, en este caso poliuretano. No se han realizado estudios de cámaras de aislamiento acústico con las combinaciones de parámetros que se muestran en este estudio.

De acuerdo a la literatura, no se han hecho estudios similares con la combinación de materiales y tamaños de la cabina sujeta al presente análisis, análisis. Sin embargo, el uso de la simulación y la aproximación con modelos propuesto y validados por la experimentación, permite una comparación entre los resultados de la simulación y los cálculos con las metodologías definidas por los autores.

\section{COMPARACIÓN CONTRA VARIOS MÉTODOS}

Se realizó la comparación de los resultados usando la ley de Masa de una pared doble a incidencia Normal, el método de Jones y dBwave para el tratamiento 1, se observa que el método de Jones en promedio da el nivel mayor de aislamiento.

Los resultados indican que se pueden variar los niveles de aislamiento, usando materiales comunes, el cálculo con dBwave se ajusta en ciertas frecuencias a los resultados de los métodos de cálculo, los cuales a veces son complicados, el uso del programa facilita

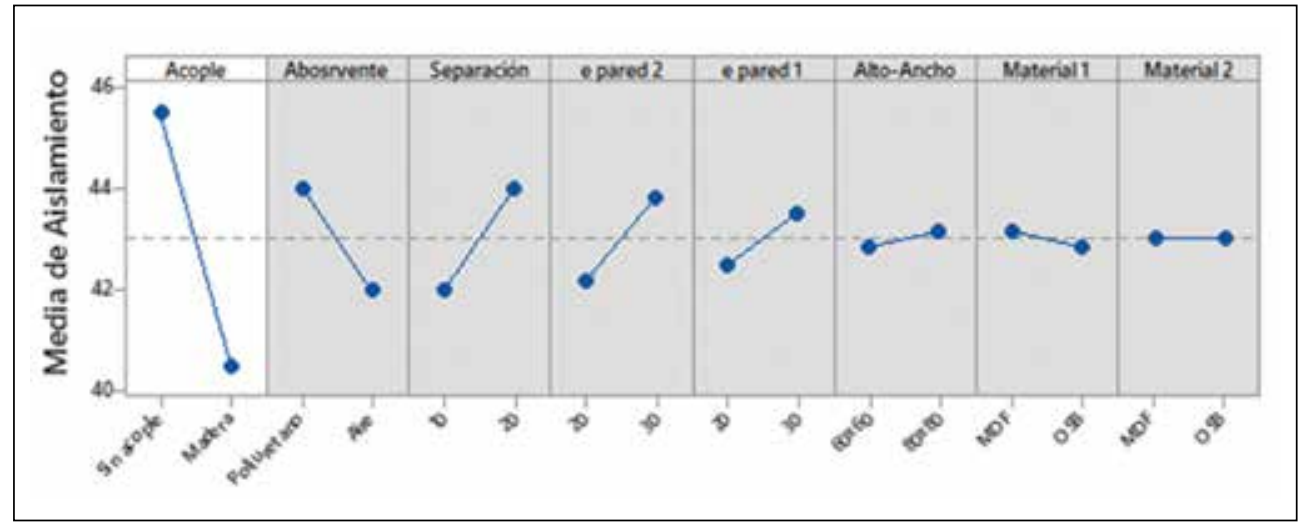

Figura 22. Gráfica de efectos principales para el aislamiento acústico. Describe cómo afectan los cambios en un solo factor a la media de aislamiento. El fondo gris representa los factores que podrían eliminarse del modelo porque no son estadísticamente significativos. 


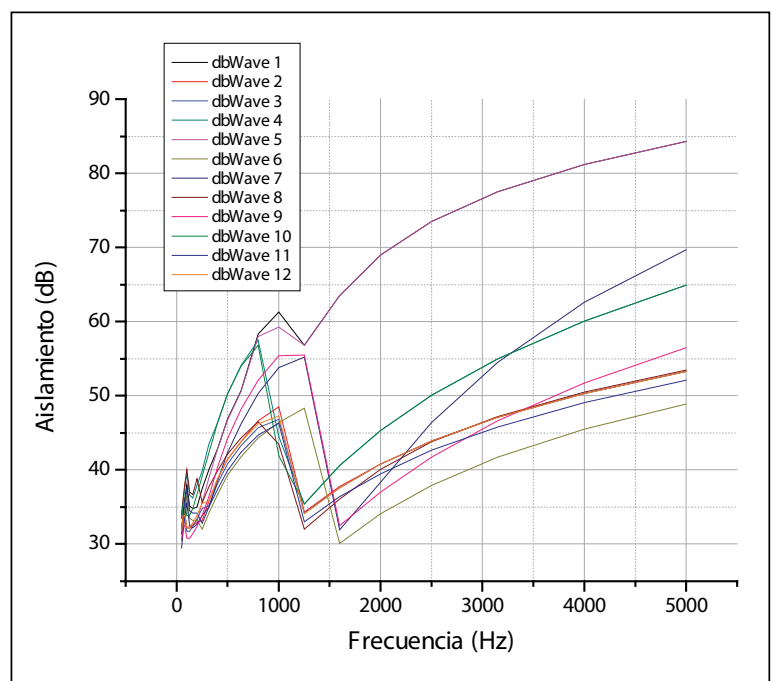

Figura 23. Resultados de la simulación en el programa dBwave para las 12 combinaciones de espesor, material y absorbente definidos en la tabla 4.
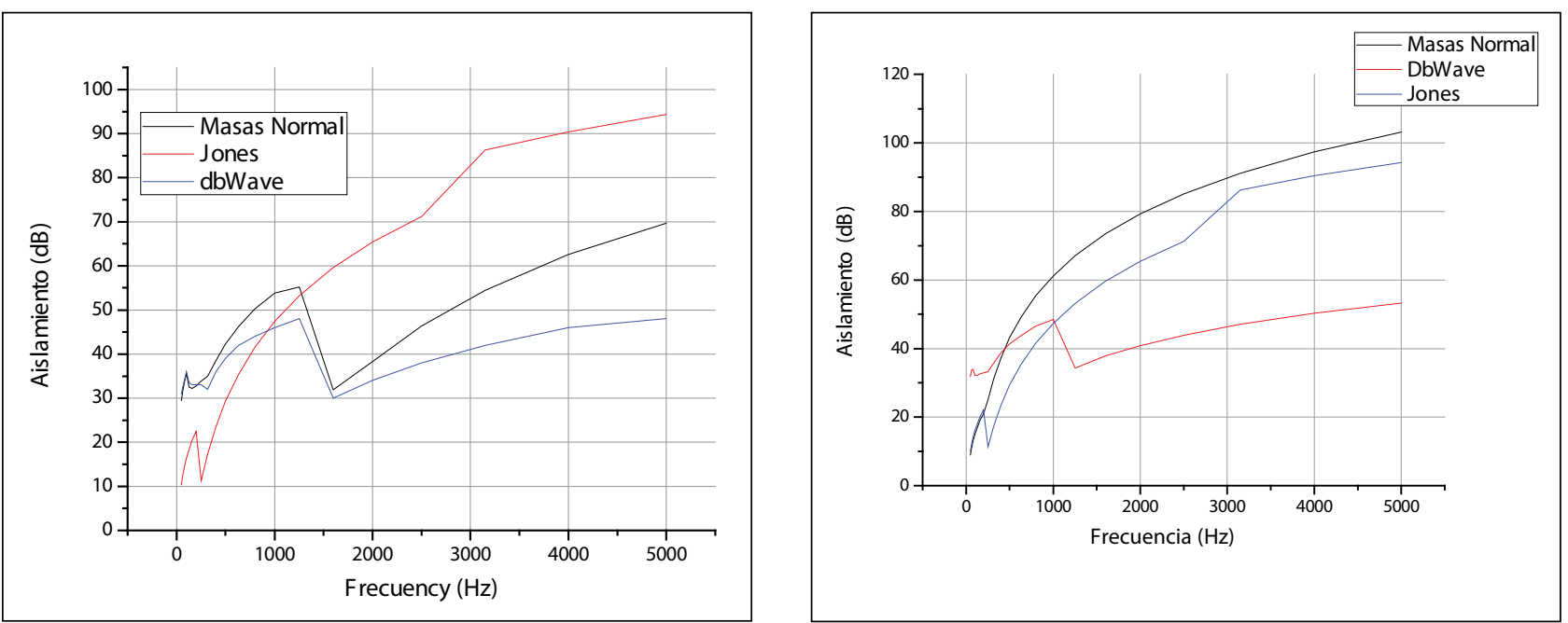

Figura 24 Comparación de los niveles de aislamiento acústico (TL) con el método de Jones, Ley de Masa de una pared doble a incidencia Normal y la simulación con dBwave para el tratamiento 1 y tratamiento 2 .

la configuración de paneles y la obtención de un resultado adecuado.

La figura 24 muestra la diferencia en el nivel de aislamiento $(\mathrm{TL})$ mediante la simulación realizada en dBwave y el método de Jones. Se puede observar que el nivel de aislamiento sólo coincide e los $2800 \mathrm{~Hz}$ y que bajo ese valor la simulación da un valor mayor, en promedio el valor adicional de aislamiento es de $22 \mathrm{~dB}$, esto puede deberse a que en el cálculo con el método de Jones no se considera el material aislante que va entre las 2 láminas.

En un estudio futuro se debe realizar la medición de los niveles de aislamiento (TL) construyendo una cabina de insonorización que cumpla los parámetros de mayor aislamiento, para validar los resultados teóricos de y de la simulación.

\section{CONCLUSIONES}

El principal factor que incide en el aislamiento acústico con los tratamientos definidos es el tipo de acople, por lo que en el diseño final de la cabina se deberá emplear el diseño sin acople, que maximiza a más de $45 \mathrm{~dB}$ el índice.

El material usado en el diseño no afecta el índice de aislamiento acústico. Se tienen niveles de aislamiento acústico similares con ambos materiales.

La combinación que sugiere se maximice el índice de aislamiento acústico es sin acople, con absorbente de poliuretano, separación de $20 \mathrm{~mm}$, espesor de las paredes de $30 \mathrm{~mm}$.

Ninguno de los tratamientos realizados cumple la combinación de parámetros que maximizaría el aislamiento acústico. Al realizar esta combinación se obtiene la siguiente curva de aislamiento. Sin embargo, el tratamiento 1 es el que da el mayor aislamiento.

El alto y ancho de las paredes no influyen en el aislamiento acústico, por lo que se tiene libertad en definir las medidas necesarias para cumplir los requisitos ergonómicos de la prueba de medición de 
ruido.

En la mayoría de los casos se produce una disminución del aislamiento acústico entre 1200 y $1800 \mathrm{~Hz}$, debido al fenómeno de coincidencia. Esta disminución en las combinaciones 1 y 5 es baja, por lo que se recomienda el tratamiento 1 para el diseño de la cabina.

De acuerdo a la literatura, no se han hecho estudios similares con la combinación de materiales y tamaños de la cabina sujeta al presente análisis, sin embargo el uso de la simulación y la aproximación con modelos propuestos y validados por la experimentación, permite una comparación entre los resultados de la simulación y los cálculos con las metodologías definidas por los autores.

\section{REFERENCIAS}

- AATEPA. (2016). "Libro blanco del poliuretano proyectado e inyectado". Madrid: AISLA, Asociación de Instaladores de Aislamiento.

- Arunkumar, M.P., J. P., K. G., M. L. (2018). "Vibro-acoustic response and sound transmission loss characteristics of truss core sandwich panel filled with foam". Aerospace Science and Technology, pp. 1-11.

- Barrionuevo, D. y Gonzales, J. (2012). "Criterios Generales para el diseño de cámaras anecoicas." Córdoba: Universidad Tecnológica Nacional.

- Dekustic (2017). Dekustik. [En línea] Available at: http://www.decustik.com/arxius/ docs/1467280372_cast_Catalogo_decustik_2016_ES.pdf

- Fu, T. y otros. (2018). "An analytical study of sound transmission through stiffened double laminated composite sandwich plates". Aerospace Science and Technology, pp. 92-104.

- Hai, Z. (2019). "A kind of soundproofed cabin suitable for automobile wind tunnel test". China, Patente $n^{\circ} \mathrm{CN} 209166834 \cup$.

- lac acoustics. (2019). https://www.industrialnoisecontrol.com. [En línea] Available at: https:// www.industrialnoisecontrol.com/products/ acoustical-test-measurement-chambers [Último acceso: 20 nov 2019].

- ISO. (1997). "Acoustics -- Sound absorbers for use in buildings -- Rating of sound absorption." s.l.: International Organization for Standardization.

- ISO. (2013). "UNE 717-1 Evaluación del aislamiento acústico en los edificios y de los elementos de construcción". Madrid: AENOR.

- Ivey, J. (2018). "Covered plastic acoustic enclosure." United States, Patente $n^{\circ}$ US20180124486A1.
- Jie, K. L. \& Huann, T. C. Y. K. Y. H. G. Y. (2017). "Numerical analysis of transmission loss through various noise barrier". Proceedings of the International Conference on Vibration, Sound and System Dynamics, pp. 60-64.

- Jingyang, C. y Wanli (2018). A kind of intelligent hearing test soundproof room. China, Patente $n^{\circ}$ CN208073037U.

- Jones, R. (1981). "Field sound insulation of load-bearing sandwich panels for housing". Noise Control Engineering, pp. 90-105.

- Lehman Fernández, C. S. (2007). "Revisión de los Algoritmos de Predicción del Aislamiento Proporcionado por Paredes Dobles: un Análisis". Valdivia: s.n.

- Llopis Reyna, A., Uris Martínez, A. y Guillen Guillamón, I. (2010). "Cálculo del índice de reducción sonora de ventanas con ruido laminar". Valencia: Dept. Física Aplicada. Universidad Politécnica de Valencia.

- Rao, G., Frank, F. y Gardonio, P. (2006). "Transmission of Sound through Partitions". En: A. Press, ed. Sound and Structural Vibration: Radiation, Transmission and Response, 2nd Edition. s.l.: s.n., pp. 277-373.

- Recuero López, M. (2000). "Ingeniería Acústica". Madrid: Paraninfo.

- Renault (2004). s.l.: Normalisation Renault Automobiles.

- Rossing, T. D. (2007). Handbook of Accoustics. Second ed. Standford: Springer.

- Sharp, B. H. (1973). "A study of techniques to increase the sound insulation of building elements". Wyle Laboratory Report No. WR 73-5.

- Shiming, B. y otros (2015). "Modular home use mini soundproof room". China, Patente $n^{\circ}$ CN204676935U.

- Supacoustic. (2006). https://supawood.com. au/supawood-products/supacoustic. [En línea] Available at: https://supawood.com.au/technical-information/acoustics [último acceso: 2110 2017].

- Systems, N. S. (2017). http://www.noisestopsystems.co.uk. [En línea] Available at: http://www. noisestopsystems.co.uk/wall-soundproofing/ nssw2-plus-soundproofing-wall-board?zenid=2d24d42dc-946c51a22cbac554e901f03

- Yamazoe, S. y otros. (2019). "Soundproof construction, soundproof enclosure and soundproof box". Japan, Patente n JP6550523B1. 